\title{
Simultaneous Treatment of Photobiomodulation and Demineralized Bone Matrix With Adipose- Derived Stem Cells Improve Bone Healing in an osteoporotic bone defect
}

\author{
Rouhallah Gazor $^{1}$, Mehrdad Asgari ${ }^{1,2,3^{*}}{ }^{(}$, Mohammad-Amin Abdollajhifar ${ }^{(\mathbb{D}}$, Pejman Kiani ${ }^{\left.5^{(}\right)}$, Fatemeh

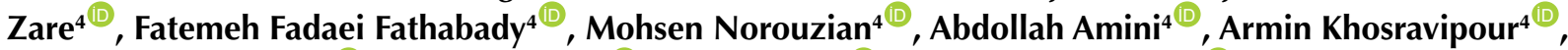 \\ Rahimeh B. Atashgah ${ }^{\circledR}$, Mahsa Kazemi ${ }^{\circledR}$, Sufan Chien ${ }^{\circledR}$, Mohammad Bayat ${ }^{4,7 * \mathbb{}}$

\begin{abstract}
'Department of Anatomy and Cell Biology, Faculty of Medicine, Guilan University of Medical Sciences, Rasht, Iran 2Department of Biology and Anatomical Sciences, School of Medicine, Shahid Beheshti University of Medical Sciences, Tehran, Iran

${ }^{3}$ Department of Anatomy, Faculty of Medicine, Guilan University of Medical Sciences, Rasht, Iran

${ }^{4}$ Department of Biology and Anatomical Sciences, School of Medicine, Shahid Beheshti University of Medical Sciences, Tehran, Iran

${ }^{5}$ Department of Neuroscience and Addiction Studies, School of Advanced Technologies in Medicine, Tehran University of Medical Science, Tehran, Iran

${ }^{6}$ Department of Pharmaceutical Biomaterials, Faculty of Pharmacy, Tehran University of Medical Sciences, Tehran, 1316943551, Iran
\end{abstract}

${ }^{7}$ Price Institute of Surgical Research, University of Louisville, and Noveratech LLC, Louisville, Kentucky; USA

\section{*Correspondence to Mohammad Bayat, Department of Biology and Anatomical Sciences, School of Medicine, Shahid Beheshti University of Medical Sciences, Tehran, Iran, Postal code: 1985717443 and Price Institute of Surgical Research, University of Louisville, and Noveratech LLC of Louisville, Louisville, Kentucky;. Email: mohbayat@ sbmu.ac.ir \\ Mehrdad Asgari, Department of Biology and Anatomical Sciences, School of Medicine, Shahid Beheshti University of Medical Sciences, Tehran, Iran; And Department of Anatomy, Faculty of Medicine, Guilan University of Medical Sciences, Rasht, Iran. Email: mehrdad_ vhf@yahoo.com}

Received: May 30, 2020 Accepted: September 23, 2020 Published online August 2, 2021

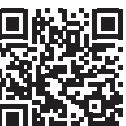

\begin{abstract}
Introduction: The ability of simultaneous treatment of critical-sized femoral defects (CSFDs) with photobiomodulation (PBM) and demineralized bone matrix (DBM) with or without seeded adipose-derived stem cells (ASCs) to induce bone reconstruction in ovariectomized induced osteoporotic $(\mathrm{OVX})$ rats was investigated.

Methods: The OVX rats with CSFD were arbitrarily separated into 6 groups: control, scaffold (S, DBM), S + PBM, S + alendronate (ALN), S + ASCs, and S + PBM + ASCs. Each group was assessed by cone beam computed tomography (CBCT) and histological examinations.

Results: In the fourth week, CBCT and histological analyses revealed that the largest volume of new bone formed in the $\mathrm{S}+\mathrm{PBM}$ and $\mathrm{S}+\mathrm{PBM}+\mathrm{ASC}$ groups. The $\mathrm{S}+\mathrm{PBM}$ treatment relative to the $S$ and $S+A L N$ treatments remarkably reduced the CSFD (Mann-Whitney test, $P=0.009$ and $P=0.01)$. Furthermore $S+P B M+$ ASCs treatment compared to the $S$ and $S+A L N$ treatments significantly decreased CSFD (Mann Whitney test, $P=0.01$ ). In the eighth week, CBCT analysis showed that extremely enhanced bone regeneration occurred in the CSFD of the S + PBM group. Moreover, the CSFD in the $\mathrm{S}+\mathrm{PBM}$ group was substantially smaller than $\mathrm{S}, \mathrm{S}+\mathrm{ALN}$ and $\mathrm{S}+$ ASCs groups (Mann Whitney test, $P=0.01, P=0.02$ and $P=0.009$ ). Histological observations showed more new bone formation in the treated CSFD of $S+$ PBM + ASCs and S + PBM groups. Conclusion: The PBM plus DBM with or without ASCs significantly enhanced bone healing in the CSFD in OVX rats compared to control, DBM alone, and ALN plus DBM groups. The PBM plus DBM with or without ASCs significantly decreased the CSFD area compared to either the solo DBM or ALN plus DBM treatments.

Keywords: Osteoporosis; Osteogenesis; Demineralized bone matrix; Human adipose-derived stem cells; Photobiomodulation.
\end{abstract}

\section{Introduction}

Osteoporosis (OP), as a global skeletal disorder, ${ }^{1}$ is characterized by the alteration of bone microstructure, reduction of bone density, robust risk of fractures, and death. OP, with 30 to $50 \%$ global privilege in postmenopausal women, is annually diagnosed in more than 9 million females in the United States. ${ }^{2}$ of different surgical treatments. ${ }^{3}$ The ovariectomyinduced osteoporosis (OVX) rat model imitates the Animal OP models are acceptable tools for the study

Please cite this article as follows: Gazor R, Asgari M, Abdollajhifar MA, Kiani P, Zare F, Fadaei Fathabady F, et al. Simultaneous treatment of photobiomodulation and demineralized bone matrix with adipose-derived stem cells improve bone healing in an osteoporotic bone defect. J Lasers Med Sci. 2021;12:e41. doi:10.34172/jlms.2021.41 
bone deterioration caused by estrogen deficiency and demonstrates the clinical signs of postmenopausal osteoporosis (PMOP). ${ }^{4}$ For this reason, the OVX rat model was commonly used in PMOP research. ${ }^{5}$

Anabolic and antiresorptive agents are approved by the Food and Drug Administration (FDA) for the prevention and treatment of OP. These medications can be associated with breast and endometrial cancers, menstruation and thromboembolic incidents ${ }^{6}$. Most of the prescribed medications for OP, such as bisphosphonates (BPs), are antiresorptive. Among the different types of BPs, alendronate (ALN) is frequently chosen. ${ }^{7}$ Antiresorptive drugs, by reducing the accessible osteoclasts, diminish bone remodeling and the risk of fracture. However, bone fragility would not be eradicated by these medications. Since microstructural degradation cannot be compensated, fragile unremodeled bones with a high degree of mineralization remain susceptible to fracture. ${ }^{8}$ Moreover, BP therapy is accompanied by some gastrointestinal troubles. ${ }^{9}$ Major problems are involved in the healing of the broken bone by OP. ${ }^{6}$

The bone graft or replacement is a necessary treatment strategy in a broad spectrum of patients with bone defects. Although the autologous bone graft is known as a gold standard treatment, additional pain at the donor site and a paucity of donor tissues have limited its applicability in patients. In this context, bone tissue engineering (BTE) is being considered as a good alternative. ${ }^{10} \mathrm{BTE}$ incorporates biomaterials, cells, and signaling factors to reconstruct bone defects. The reconstruction process is as follows: (a) cell harvest and scaffold construction. (b) Seeding the cells into the scaffold creates a "cell/scaffold" structure. Following $(c)$ structure implantation, $(d)$ generation of new bone will increase and scaffold will degenerate, finally, (e) bone reconstruction will be completed. ${ }^{11}$

Bone scaffolds not only provide a $3 \mathrm{D}$ microstructure for tissue regeneration, differentiation, nutrition, and metabolism but also supply a boosted mechanical strength. ${ }^{12}$ Demineralized bone matrix (DBM) is a bone graft extender produced from human corpse bone. ${ }^{13}$ It is a decalcified product that uses an acidic solution to remove mineral components. ${ }^{14}$ Spongy structure and flexibility (in the hydrated state) are essential characteristics of DBM scaffolds consisting of collagen (mostly type I and a few types IV and X), non-collagenous proteins, some osteoinductive growth factors (e.g. bone morphogenic proteins, BMP), and varying percentages of calcium phosphate..$^{15}$ After decalcification, BMP could be discharged from the around mineral elements and fully exert its osteoinductive capacity. The remaining DBM collagen proteins may supply a 3D structure for the ingrowth of host capillaries, perivascular tissue, and osteoprogenitor cells into the graft. ${ }^{14}$ Increasing data have shown that DBM scaffolds can increase new bone formation and enhance in vivo healing of clinical bone defects. ${ }^{16,17}$ Though DBM scaffolds have osteoinductive and osteoconductive properties, their osteoinductivity is often reduced by manufacturing processes, such as chemical decellularization and demineralization. ${ }^{18}$ Some studies have shown that DBM scaffold possesses only a low osteoinductive capacity. ${ }^{19,20} \mathrm{~A}$ hybrid allograft of the seeded bone matrix with mesenchymal stem cells (MSCs) may be suggested as a method for enhancing osteoinductivity by releasing growth factors. ${ }^{21}$ Schubert et al showed that osteogenic-differentiated adipose-derived stem cells (ASCs) are the best candidate to improve the angio-/osteogenicity of decellularized bone allografts. ${ }^{21}$

The usage of MSCs is an important element in BTE. In addition to the usage of bone marrow-derived mesenchymal stem cells (BMMSCs), ASCs have recently been targeted in the BTE because of a high proliferation rate, strong osteogenic potential, and noninvasive sampling methods..$^{22}$ ASCs, similar to BMMSCs, can improve angiogenesis. ${ }^{23}$ Several experiments have shown the beneficial potential of ASCs to promote bone repair in the various animal models of femoral and skull criticalsized defects. ${ }^{24-26}$ Although ASCs are reviewed as a successful bone healing strategy, ${ }^{27}$ there are some issues that should be addressed. The low viability of cells after implantation has attracted substantial attention. Hence, enhancing the survival of transplanted ASCs is crucial for increasing the success rate of ASC-based treatment. ${ }^{28}$ The interplay between cells and scaffold structure can facilitate restorative osteogenesis by increasing the viability of stem cells and guiding the differentiation of stem cells toward the bone matrix formation. ${ }^{22}$

Photobiomodulation (PBM), as a treatment strategy, is expected to resolve the weak potential of transplantation and viability of ASC. ${ }^{29}$ MSCs and progenitor cells are particularly susceptible to PBM. ${ }^{30}$ It has been shown that PBM potentially promotes the proliferation, differentiation, and growth factor secretion of MSCs through altering gene expression and activation. ${ }^{31}$ PBM is effective in various clinical fields such as pain attenuation, wound healing, bone healing and bone remodeling. ${ }^{32}$ Researches have also indicated that PBM impacts osteogenesis and bone regeneration in comparable animal studies. ${ }^{33}$ PBM hastens bone healing and remodeling mainly by enhancing the deposition of hydroxyapatite and collagen in the osteoid matrix, the mechanical qualities of the bone, vascular proliferation, gene expression and protein production in the bone. ${ }^{34}$

Hybrid human[h] ASCs-hDBM osteoconductive scaffolds in bone regeneration have been successfully applied in previous experiments. ${ }^{22,35-37}$ Moreover, Schubert et al showed that osteogenic-differentiated ASCs are the best candidates to improve the angio-/osteogenicity of decellularized bone allografts. ${ }^{38}$ This research aimed to test the impact of simultaneous treatment of PBM and hybrid BTE scaffolds (including hDBM, and hASC) on 
accelerating the healing rate of a critical-sized femoral defect (CSFD) in an OVX rat model.

\section{Materials and Methods}

\section{Animals and Study Design}

Seventy-two female Wister rats (3 months old, 200-250 g) were used in this research. The animals were caged under environment-controlled conditions (12-hour light/dark cycle, $22-25^{\circ} \mathrm{C}$ ). A standard laboratory diet (Behparvar, Tehran, Iran) and tap water were available ad libitum. The animals were anesthetized by the intraperitoneal administration of xylazine and ketamine and then subjected to OVX. ${ }^{40}$ Bilateral CSFD surgeries were done 14 weeks later.

The rats were randomly divided into six groups (each group, $n=6$ rats). (1) In the control group (C), CSFDs were not treated. DBM was transplanted into the CSFD in groups 2-6 (DBM-CSFD). (2) S group, (DBM-CSFD). (3) $\mathrm{S}+$ PBM group, the DBM-CSFDs were treated with PBM. (4) S + ALN group, the DBM-CSFDs were treated with $0.01 \mathrm{mg} / \mathrm{kg} / \mathrm{d}$ ALN subcutaneously. ${ }^{39}$ (5) S + ASCs group, ASCs were seeded into DBM and then implanted into the CSFD. And (6) S + PBM + ASCs group. ${ }^{41}$ At the fourth (anabolic phase of bone healing) and eighth week (catabolic phase of bone healing) post-operation, six rats from each group were euthanized and the left and right distal femurs were isolated and CSFDs were examined by the cone beam computed tomography (CBCT) scan and histology respectively. Groups 2-6 were considered as treatment groups.

\section{Procedure for Ovariectomy}

Bilateral OVX was conducted on female rats according to a previously described method. In brief, after exposure of abdominal cavity, 2 ovaries were exposed and excised from the rats under sterile condition and general anesthesia. The rats were housed for 14 weeks in order to establish OP. Ceftriaxone, as the antibiotic therapy, was administered. ${ }^{40}$

\section{Preparation of the Demineralized Bone Matrix Scaffold} The DBM was a donation from Hamanand Saz Baft Kish Company (TRC Corporation, Kish, Iran). It was prepared from the cancellous sections taken from the diaphysis of the long bones of human cadavers under sterile conditions. After cutting the bones into the small pieces, a series of physical and chemical procedures were performed to remove mineral content, blood, fat and cells. ${ }^{41}$

Isolation, Expansion, and Immunophenotyping of Human ASCs

hASCs were purchased from Royan cell bank institute (code: RSCB:0591). The flow cytometry analysis was performed to characterize the hASCs based on previously reported MSC markers. ${ }^{41,42}$

\section{Seeding hASCs on DBM Scaffolds}

The DBM scaffolds were cut into cubes $(3 \mathrm{~mm}$ in diameter to $5 \mathrm{~mm}$ in thickness) and transferred into 24-well plates. Each DBM scaffold was incubated with $10 \mu \mathrm{L}$ of enriched hASCs (passage $4,3 \times 10^{4} / \mu \mathrm{L}$ density) for 2 hours. Then $150 \mu \mathrm{L}$ of the culture medium was added to each well. The wells were incubated at $37^{\circ} \mathrm{C}$ and $5 \% \mathrm{CO} 2$ for 24 hours to allow cell adhesion. Finally, each cube of the DBM (containing hASCs) was implanted into the CSFD of the rats (groups 5, and 6). ${ }^{41}$

\section{CSFD Model and DBM Scaffold Transplantation}

Fourteen weeks after the OVX surgery, the rats were anesthetized by intraperitoneal injections of ketamine $(50 \mathrm{mg} / \mathrm{kg}$ ) $)$ and xylazine $\left(5 \mathrm{mg} / \mathrm{kg}\right.$,). ${ }^{43}$ Standardized $3 \mathrm{~mm}$ femoral cylindrical defects were drilled in the distal metaphysis of the femurs of both sides ( 2 defects in each rat). The defects in groups 2-4 were filled with the DBM scaffold. In the S + ASCs and S + PBM + ASCs groups, the bone defect was filled with the hybrid hASCs -DBM scaffold. ${ }^{41}$

\section{Photobiomodulation}

CSFD in groups 3 and 6 were immediately subjected to PBM (MUSTANG 2000, LO7 probe; Technica Co, Moscow, Russia) after surgery. PBM therapy continued 3 days a week for 4 and 8 weeks on the basis of the time of the euthanization. ${ }^{41}$ The rats were sedated with half dosages of anesthetizing drugs which were used for general anesthesia. ${ }^{43} \mathrm{PBM}$ was performed in each session at three points; one point was exactly over the CSFD, the second point was $1 \mathrm{~cm}$ proximal to the center of the CSFD, and the third point was $1 \mathrm{~cm}$ distal to the center of the CSFD. The PBM parameters are shown in Table $1 .{ }^{41,44}$

\section{CBCT Imaging and Measurement of the Bone Defect Area}

Four and 8 weeks after implantation, the rats were sacrificed. The removed femora were fixed in $10 \%$ formalin saline for 48 hours and subjected to the CBCT imaging system (PaX-i3D Green of Vatech scanner, Gyeonggi-do, Korea). During bone defect scanning, 50 $\mathrm{kVp}, 2 \mathrm{~mA}, 0.3 \mathrm{~mm}$ voxel size and $50 \times 50 \mathrm{~mm}$ field of view were used. The scanned slices were reconstructed into DICOM files. The bone defect area of CSFDs in the studied groups was measured. The bone defect area (\%) was calculated according to the residual defect area on a given week $(\mathrm{tx})$ relative to the measured defect area on the day of surgery $(3 \mathrm{~mm})$ as follows:

Bone defect area $=(\mathrm{tx}$ defect area $) /($ original defect area $)$ $\times 100$

\section{Histological Examination}

After CBCT scanning, the femurs were decalcified in $10 \%$ EDTA for two weeks, and the EDTA solution was changed 
Table 1. Photobiomodulation parameters in the study.

\begin{tabular}{lc}
\hline Parameters & Dose and unit \\
\hline Peak power output & $75 \mathrm{w}$ \\
Average power & 0.001 \\
Wavelength & $890 \mathrm{~nm}$ \\
Pulsed frequency & $80 \mathrm{~Hz}$ \\
Spot size & $1 \mathrm{~cm}^{2}$ \\
Pulsed duration & $180 \mathrm{~ns}$ \\
$\begin{array}{l}\text { Duration of exposure of each } \\
\text { point }\end{array}$ & $900 \mathrm{~seconds}$ \\
Energy density for 1 shooting, & $0.972\left(\mathrm{~J} / \mathrm{cm}^{2}\right), 2.96\left(\mathrm{~J} / \mathrm{cm}^{2}\right)$, \\
1 session(1day), 1week, & $9.74\left(\mathrm{~J} / \mathrm{cm}^{2}\right), 34.99\left(\mathrm{~J} / \mathrm{cm}^{2}\right)$, \\
4 weeks, and 8weeks & $69.98\left(\mathrm{~J} / \mathrm{cm}^{2}\right)$. \\
\hline
\end{tabular}

twice a week. Sections with $5 \mu \mathrm{m}$ thicknesses were cut with a microtome and stained with the hematoxylin and eosin (H\&E) method. New bone formation at the bone defects was observed and examined descriptively by an optical light microscope (Olympus, Japan). ${ }^{45}$

Prior to cell transplantation, the hASCs were marked with 1,1'- dioctadecyl-3,3,3',3' - tetramethyl indocarbocyanine perchlorate (DiI, Invitrogen)46. The hASCs were ready by a standard technique. Initially, $50 \mu \mathrm{g}$ of DiI was added to $50 \mathrm{~mL}$ of DMSO. Then, $1 \times 10^{6}$ cells were exposed to $5 \mu \mathrm{L}$ of DiI per $\mathrm{mL}$ of the medium at $37^{\circ} \mathrm{C}$ for $30 \mathrm{~min}$ and $4{ }^{\circ} \mathrm{C}$ for 15 minutes. Next, the cell suspension was centrifuged, the media was eliminated, and the cells were twice washed in sterile PBS. Subsequently, DiI-marked hASCs were seeded into the DBM scaffolds and checked to utilize a fluorescent magnifying lens (Olympus, Tokyo, Japan). At four and eight weeks after transplantation of the marked hASCs, the CSFDs were removed, and histological sections of the CSFD were ready and stained with the H\&E strategy. The color intensity of the DiIpositive region in the fracture bed of $\mathrm{S}+\mathrm{ASC}$ and $\mathrm{S}+$ $\mathrm{PBM}+\mathrm{ASC}$ groups was calculated utilizing the Image $\mathrm{J}$ program. ${ }^{46}$

\section{Statistical Analysis}

The data are presented as mean \pm standard deviation (SD). The normal distribution of the data was analyzed by the Shapiro test. One-way analysis of variance (ANOVA) with the least significant difference (LSD) post-hoc test was applied. A $P$ value of $<0.05$ was considered statistically significant. The Mann-Whitney $U$ test was used for statistical analysis of non-parametric data.

\section{Results \\ hASCs Stem Cell Surface Marker Characterization}

One week after the initial isolation of hASCs, the flasks were filled with cells. Spindle-shaped adherent cells were observed at passage 4 (Figure 1A). To examine the cell phenotype, passage 4 of the hASCs was characterized. Characterization of cell surface marker by flow cytometry indicated that a high percentage of hASCs (Figure 1B-E) were positive for CD105 (84.8\%) and CD73 (99.8\%) as markers of MSCs. The percentages of positive cells for hematopoietic markers including CD45 (0.4\%) and CD31 $(0.3 \%)$ were low. The evaluated surface markers indicated the true stemness of hASCs population.

\section{DiI Labeling}

Figure 2 displays the magnified micrographs of sagittal sections of stained H\&E specimens obtained from the $S$ + ASCs and S + PBM + ASCs groups 4 and 8 weeks post-

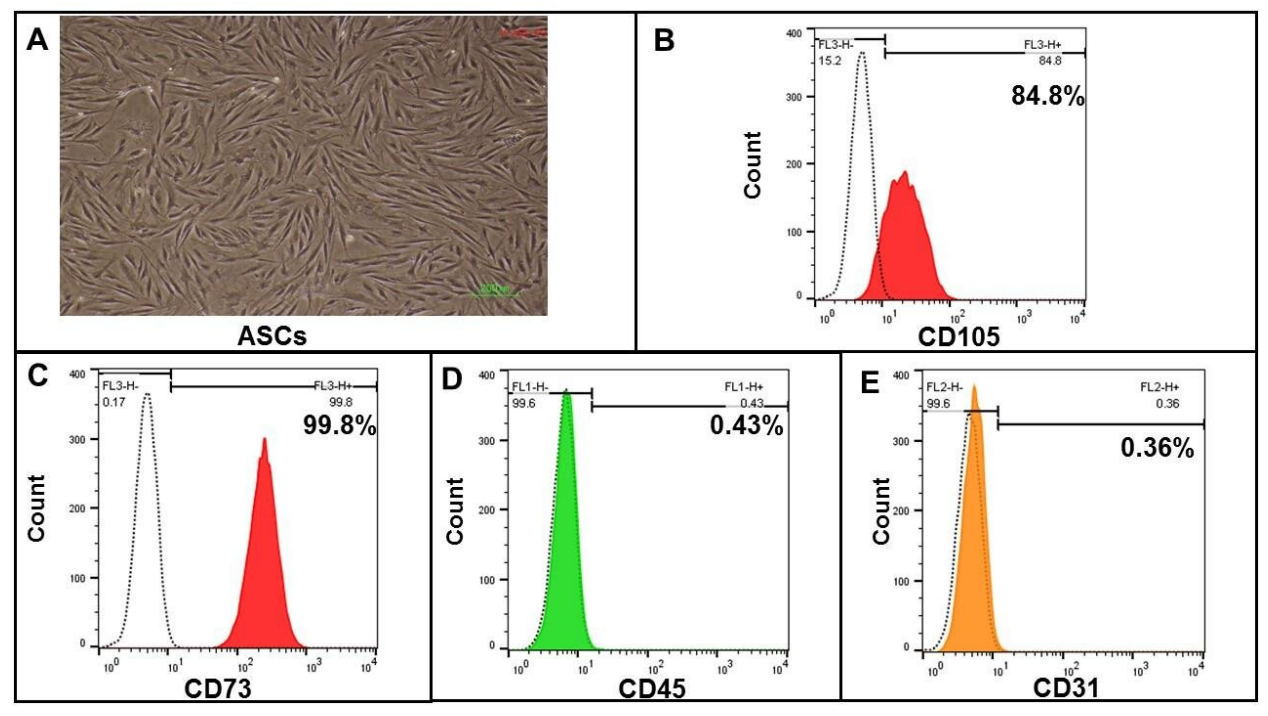

Figure 1. Identification of hASCs. (A). hASCs at Passage-4 sat 100×magnification under the inverted microscope (B). hASCs were positive for CD105 (C) and CD73 (D) as MSCs markers. Low percentages of cells were positive for CD45 (D) and CD31 (E) as hematopoietic markers. 


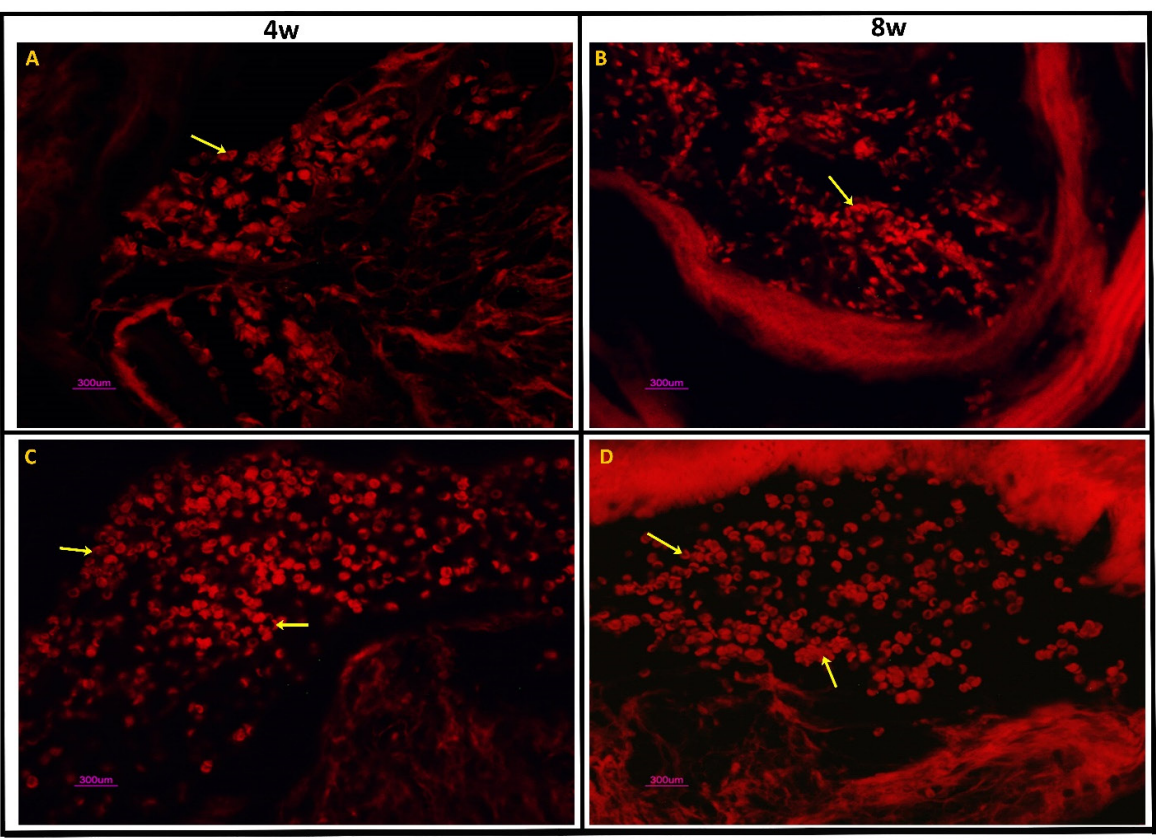

Figure 2. Imaging of hASCs Migration in CSFD. Sections of paraffin-embedded tissue were prepared at 4 and 8 weeks following the transplantation

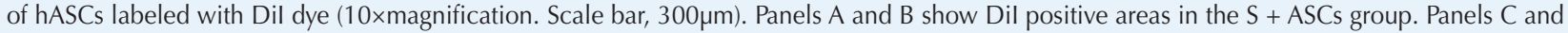
$\mathrm{D}$ show Dil positive area in group $\mathrm{S}+\mathrm{PBM}+\mathrm{ASC}$.

implantation. In the histological sections, numerous DiIpositive (red) cells were identified throughout the defect (Figure 2A-D). The results indicated that the implanted hASCs survived throughout the experimental time and migrated to the defect.

\section{Outcomes of CBCT Evaluation}

The CBCT imaging at the fourth and eighth weeks after bone defect induction revealed that the bone defect mostly remained empty in the control group. It also illustrated poor bone healing and shattered bone structure near the implantation site in the S, S + ALN and S + ASCs groups (Figure 3A). It seems that applied treatment strategies in these groups failed to heal the defects. In contrast, the $S+$ $\mathrm{PBM}+\mathrm{ASCs}$ group triggered new bone formation in the fourth week after surgery. In this group, a large area of the femur recovered and only small defect lesions remained in the eighth week after surgery. The S + PBM group not only elicited abundant new bone formation in the fourth week after surgery, but also almost filled the defect in the eighth week after surgery. As is shown in Figure 3b, the three-dimensional CT images of CSFD showed that the greatest amount of new bone formation occurred in the $\mathrm{S}+\mathrm{PBM}$ and $\mathrm{S}+\mathrm{PBM}+$ ASCs groups in the fourth and eighth weeks after surgery. The $S+P B M$ group regenerated the highest amounts of mineralized bone in the eighth week after surgery.

Figure 4 shows the percent of the defect area. At the fourth week post-surgery, the treatment groups (2-6) compared to the control group showed a significant decrease in the defect area (Mann-Whitney test, all $P=$ 0.008). $S+P B M$ treatment significantly decreased the defect area compared with the S and S + ALN treatments (Mann-Whitney test, $P=0.009$ and $P=0.01$ ). $S+P B M$ + ASCs treatment significantly decreased the defect area compared with the S and S + ALN treatments (MannWhitney test, $P=0.01$ ) (Figure 4, Panel A).

At the eighth week post-surgery, the control group showed a significant increase in the defect area compared with the treatment groups (Mann-Whitney test, $P=0.01, P=$ $0.009, P=0.03, P=0.02$ and $P=0.009$, respectively). The defect area in the $S+P B M$ treatment was significantly smaller than the $\mathrm{S}, \mathrm{S}+\mathrm{ALN}$ and $\mathrm{S}+$ ASCs treatments (Mann-Whitney test, $P=0.01, P=0.02$ and $P=0.009$ ) (Figure 4B). While the defect area in the S + PBM + ASCs group reached $0.9 \mathrm{~mm}$, it subsided to $0.5 \mathrm{~mm}$ in the $S+$ PBM group (Figure 4C).

\section{Histological Findings}

At the 4 and 8 weeks after surgery, while defects were filled with fibrous tissue in the control group, at the same time the newly formed bone was observed in the treatment groups. The extent of new bone formation in the S PBM, $\mathrm{S}+\mathrm{ASC}$ and $\mathrm{S}+\mathrm{PBM}+\mathrm{ASC}$ groups was greater than the control group. Compared to the other treatment groups, the defects of the $S+$ PBM group exhibited much more new bone formation and many defects had disappeared. Moreover, the defect areas exhibited maximum bone formation (from the defect margin to the center), remodeling and consolidation in the $\mathrm{S}+\mathrm{PBM}+\mathrm{ASCs}$ 


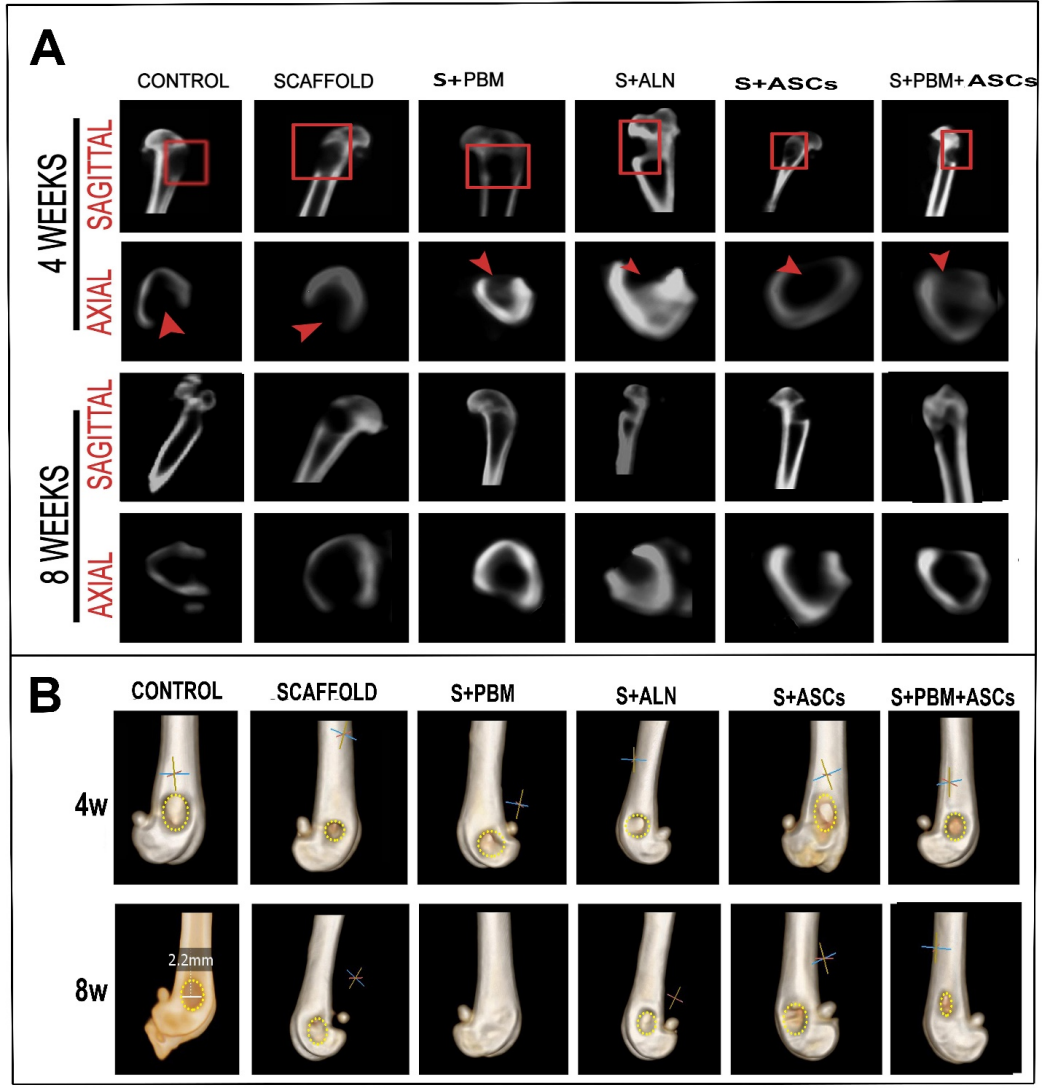

Figure 3. Bone Healing as Evaluated by СВCT. A: СВCT Images of the defect area in the 4th and 8th weeks. B: frontal view of reconstructed three-dimensional CT images of rat femurs in different groups. The open arrowheads indicate the defect regions. $n=5$ in each group.
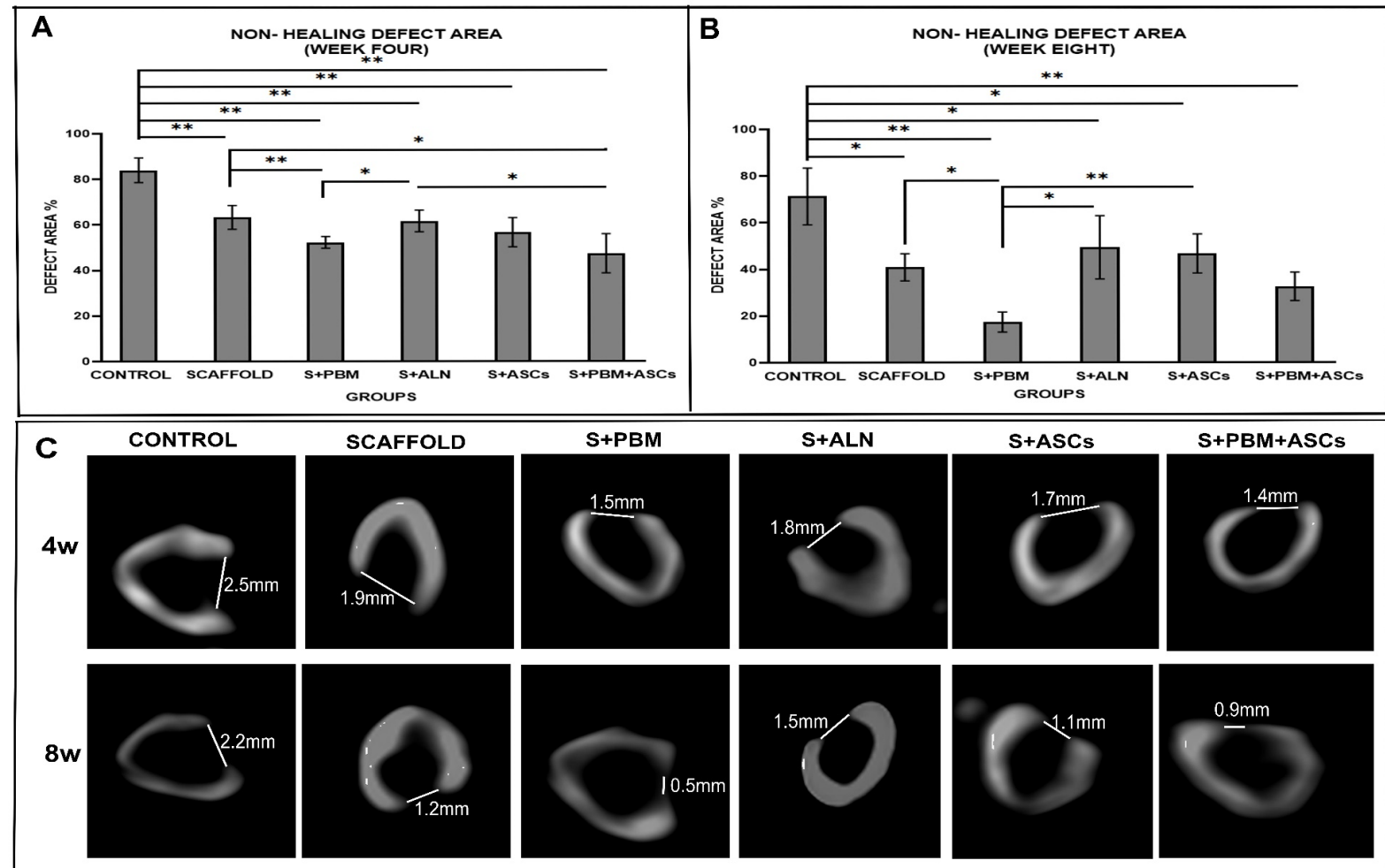

Figure 4. A-B: Comparison of the defect area percentages in the experimental groups. C: Measurement of the defect area as assessed by CBCT. Mann-Whitney test; ${ }^{*} P<0.05$ and ${ }^{*} * P<0.01$. 

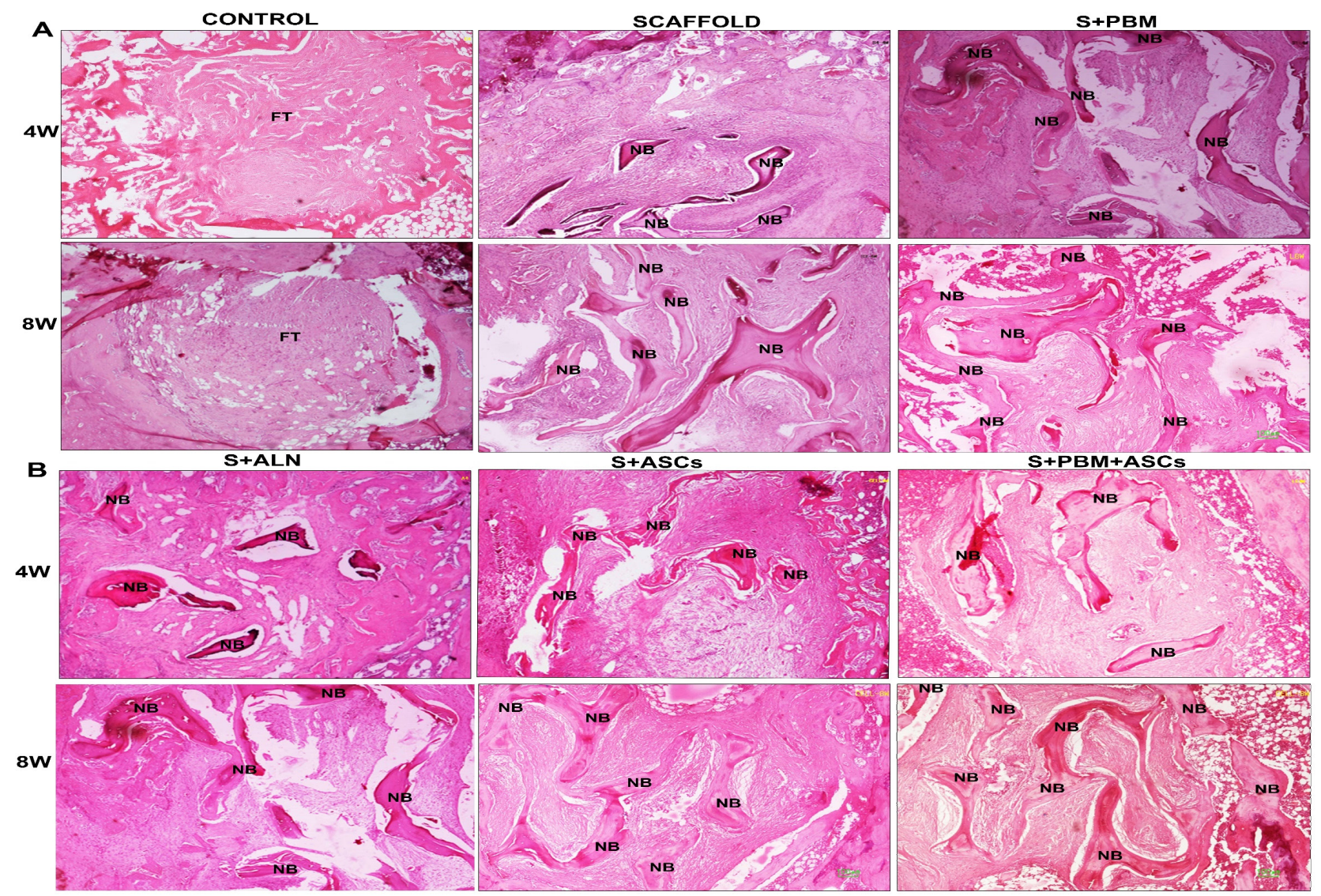

Figure 5. Photomicrographs of CSFD From Each Group at 4 and 8 Weeks (W) After Surgery; H/E staining; original magnification, X4. The image of the control group shows that most parts of the hole persisted in the fourth and eighth weeks after surgery. In both $\mathrm{S}+\mathrm{PBM}$ and $\mathrm{S}$ + PBM + ASCs groups, the hole was almost restored by the new bone formation in the eighth week after transplantation. FT, fibrous tissue; NB, new bone; S, scaffold; PBM, photobiomodulation; ASCs, adipose-derived stem cells.

group, compared to the other ones (Figure 5).

\section{Discussion}

The design of new innovations and instruments, by providing patient comfort and improving care outcomes in less time, has made the medical procedures easier to be carried out. ${ }^{47}$ Our present results demonstrate the positive impacts of the combination of PBM and DBM with or without ASCs on inducing bone regeneration and improving bone repair.

Autogenous bone grafting is known as the gold standard for healing bone fractures. Meanwhile, certain drawbacks such as donor site morbidity and insufficient bone resources cannot be resolved. ${ }^{48}$ BTE techniques, including osteogenic cells, scaffolds, and either external or in situ biostimulators (signaling) such as PBM, are very encouraging as a solution for the management of nonhealing large bone defects. Hence, they have recently been introduced as an alternative to conventional treatments. BTE techniques show huge potential for stimulating bone repair and overwhelming some of the disadvantages of bone grafting. ${ }^{49}$ Seebach et al compared various biological and synthetic scaffolds for choosing a suitable one in bone replacement therapy. Intriguingly, bone allografts relative to all other scaffolds displayed the highest cell conductivity probably due to the collagen-fiber structure. ${ }^{50} \mathrm{DBM}$ has long been applied for bone transplantation. BMPs and other growth factors in the DBM make it capable to induce osteogenesis. During bone repair, BMPs play an essential role in the development and differentiation of several types of cells. They recruit the MSCs to the healing site and stimulate their alteration to a bone-forming lineage. ${ }^{35}$ The osteoinductivity of DBM relies on the BMP-2 and BMP-7 levels as the key growth factors. Variability in the osteoinductive potential of human DBM products is due to different extraction and processing procedures of DBM that can reduce the level of BMP in the sterilization and storage procedures. Whereas the product is subject to the same procedure, the difference depends on the quality of allogeneic bone donors. In terms of therapeutic application and effectiveness, although DBM has some prospects, confirmative evidence to support its singular use as a bone substitute is restricted.$^{51}$ Hence, PBM and BTE offer an opportunity to improve the osseointegration of DBM. We aimed to increase the osteogenic properties of DBM by BTE and PBM approaches to treat femoral 
bone defects.

Postmenopausal women with OP have a high risk of fracture. Several drugs have been used for the treatment and prevention of OP and fractures. The side effects of medications to treat OP tend to reduce the life quality in affected persons. ${ }^{52} \mathrm{BT}$ is the gold standard for restricting bone loss. The bone loss that occurs in numerous conditions such as age-related OP in both males and females is characterized by increased osteoclast-mediated bone absorption..$^{53}$ Although radiographic and histological data revealed that the application of ALN plus DBM treatment improved bone healing relative to the control group, no differences were observed between the ALN plus DBM treatment and other therapeutic groups. These conflicting data could be explained by the fact that longterm BT might actually inhibit bone remodeling to such an extent that it disturbs the normal healing of the bone and increases the risk of fracture. ${ }^{54} \mathrm{~A}$ research by Kennel et al showed that negative impacts of BT (such as ALN) might restrict $\mathrm{BT}$ prescription for $\mathrm{OP}$ in some patients and best usage of BT for OP needs suitable calcium and vitamin $\mathrm{D}$ intake. ${ }^{55}$ Accordingly, the lengthy treatment of OP cases with nitrogen-including BT has been linked with uncommon fractures in the femur. ${ }^{56} \mathrm{ALN}$, which is often prescribed for OP, has been linked with various adverse effects in different organs of the human body, such as gastrointestinal, endocrine, and musculoskeletal systems. ${ }^{57}$

MSCs have been demonstrated to be the most appropriate stem cells for bone regeneration. In addition to BMMSCs, the applicability of ASCs in the BTE has been rising in the previous years. ${ }^{22}$ It is worth noting that BMMSC sampling is quite invasive and painful, with possible morbidity and poor outputs. On the contrary, ASCs sampling raises outputs and decreases the pain in a simple procedure. Adipose tissue also contains considerably more stem cells than bone marrow (5\% vs. $0.01 \%) .^{58}$ Various studies have documented that ASCs relative to BMMSCs may have equal or even superior regenerative capacity (1) due to a higher cell proliferation rate (2), higher regenerative factor secretion (3) and higher immune suppression capacity. ${ }^{59}$ In different animal studies, such as rat and canine models of skull defects, femoral head osteonecrosis, femur defect, disruption osteogenesis, and spine fusion, the osteogenic ability of ASCs was demonstrated alone or in combination with a scaffold. Different scaffolds may be utilized for ASCs seeding, involving apatite-coated poly (lactic-co-glycolic acid) scaffolds, collagen-ceramic runners, type I collagen matrix and coral scaffolds. ${ }^{60}$ To date, animal studies to repair bone defects have used scaffold transplantation, cell transplantation and hybrid scaffold-cell transplantation. In addition, the transplantation of a hybrid scaffold-cell structure has been shown to have greater osteogenic ability than that achieved by co-transplantation of scaffolds and
MSCs. Important considerations in the transplantation of MSCs involve how the cells can be transferred to the surgical site or how the inserted cells can be functionally grafted onto it. It is believed that isolated MSCs cannot themselves be fixed and readily stay in the region of a bone defect. Thus, the MSCs must be added along with any carriers or scaffolds. ${ }^{61}$

Our findings revealed that the treatment of CSFD with ASCs-seeded DBM scaffolds (S+ASCs group) significantly decreased the defect area relative to the control group. This analysis revealed the significance of ASCs in bone healing. Kloeters et al, in an experiment, showed the pro-osteogenic impact of ASC-seeded bone allografts in rabbits. ${ }^{61}$ Schubert et al investigated that bone allografts were colonized with ASCs and BMMSCs in a murine ectopic transplantation model. ${ }^{21}$ Various experiments on the ASCs, including co-culture with bone allografts or synthetic scaffolds, demonstrated an increase in osteogenesis and angiogenesis potentials of hybrid scaffold..$^{2-4}$ In terms of histological analysis, the S+ASCs treatment displayed more new bone formation relative to the control group. However, The CBCT images showed that treatment with ASC-seeded DBM scaffolds failed to completely fill the defect area. This result indicated that ASC-seeded DBM scaffolds can form new bone in a small area of femoral defects. Nevertheless, the number of migratory cells might be not adequate to allow new bone to cover the CSFD completely.

Recent studies have demonstrated a substantial decline in survivable MSC numbers in animal models of skin injury and skull defects 14 days after implantation. ${ }^{63,64}$ The main reason for unacceptable clinical outcomes of ASCs transplantation may be either the poor biological activity or the poor survival ability of injected ASCs. ${ }^{63}$ There are also attempts to apply new approaches in improving the potentials of survival, homing to the inflammation site, and immunomodulation. To our knowledge, the simultaneous healing effect of PBM and hybrid ASCsseeded DBM on patients with CSFD or animals with OP has not been reported yet.

Various experiments have indicated that PBM, by increasing the number of differentiated osteoblastic cells, enhances osteoid tissue and bone formation. It is effective through inducing osteogenic cell proliferation, modifying the activity of osteocytes and inducing the differentiation of precursor cells. ${ }^{33}$ Accordingly, our findings showed that treatment with $\mathrm{PBM}$ and ASCs-seeded DBM relative to other treatments accelerated the healing rate of CSFD in rats as analyzed by the H\&E. Incremental bone healing after PBM treatment was also observed in the CBCT analyses. The simultaneous treatment with PBM and hASC-seeded DBM demonstrated a statistically significant reduction of the defect area compared to either the solo DBM or ALN plus DBM treatments at the fourth week (Figure 4A). Some experiments have 
shown that PBM can stimulate either the mesenchymal cells indirectly or osteoblasts directly, which may lead to decreased bone mass deposition and secretion of matrix elements in the bone defects and fractures. ${ }^{65,66}$ Choi et al declared that the use of ASCs in an acellular dermal matrix concomitant with PBM exposure can substantially enhance bone formation and drastically increase the mineral density and mass volume of the bone. ${ }^{67}$

Interestingly, we found that the simultaneous administration of PBM and DBM hastened the bone healing of CSFD in the OP rats. Additionally, the CBCT photographs revealed that the simultaneous treatment of CSFD with PBM and DBM not only elicited abundant new bone formation in the fourth week after surgery, but also filled the defect in the eighth week after surgery (Figure $3 \mathrm{~A})$. It was noted that owing to its effects, PBM helps the process of new bone formation and reduces the healing time of the defects. Bosco et al demonstrated that PBM with or without the bovine bone graft could improve bone formation in rats with critical-sized calvarial defects. ${ }^{68}$ Cunha et al found that PBM improved the repair of bone defects. ${ }^{69}$ Fernandes et al demonstrated that Biosilicate $\%$ PLGA composites in association with PBM exposure by increasing the rate of material dissolution can increase the amount of newly formed bone tissue at the site of defects. ${ }^{70}$ Consistent with these previous findings, we showed that the simultaneous application of PBM and DBM resulted in an accelerated reduction in the defect area (Figure 4B-C). The increased deposition of hydroxyapatite and collagen in the osteoid matrix at the site of CSFD might be responsible for improving the healing rate of the bone. The experimental $S+P B M$ group relative to $S, S+A L N$ and $S+$ ASCs groups showed smaller defect areas at both times, mainly at the eighth week (Figure 4B). This result suggests that $\mathrm{PBM}$ increases the osteoconductive potential of biomaterials. Considering the osteoinductive properties of biomaterial and PBM, it can be postulated as an addictive action. Other authors have reported that the use of biomaterials in laser therapy can enhance the effectiveness of light. ${ }^{63}$

The present study had some limitations. First, we did not verify the optimal quantity of seeded ASCs to affect the bone repair. For example, little implanted cells might leave a dead space or cause other problems. Second, we did not verify the abilities of ASCs seeded DBM or PBM in a bone fracture model, which is relevant as more cases of bone fracture are encountered in clinical practice than of bone defects. Third, we did not demonstrate the efficacy of PBM and BTE approaches in a larger animal or a more massive bone defect model. Another limiting factor for our study was the unavailability of the micro-CT; thus, we used CBCT for evaluating the healing of bone defects.

From a clinical point of view, regeneration of $80 \%$ or more within a certain time should be considered necessary for applying a BTE as an alternative to autologous bone transfer. This remains quite a challenge, especially for large-sized defects. ${ }^{22}$ In the present study, CT scan evaluation revealed that the percentage of defect areas in the simultaneous PBM and hASC-seeded DBM treatment reached up to $32.5 \%$, whereas treated defects with PBM and DBM decreased to $17.3 \%$ (Figure 4C). On the other hand, the percentage of healed areas in the $S$ + PBM group at the 8 th week was $82.7 \%$. The present study demonstrated the therapeutic potential of PBM and DBM without cell seeding in the animal model of long bone defects. Accordingly we found that ASC, and PBM significantly increased healed bone strength in an experimental model of DBM-treated CSFD in the catabolic phase of bone healing in osteoporotic rats. ${ }^{41}$ However, ALN alone and PBM plus ADS were superior to the other protocols.

The present study demonstrated that the simultaneous hDBM + PBM treatment of CSFD significantly enhances the bone healing process in the OVX rats. Although several issues including biomechanical and molecular mechanisms of hDBM + PBM effectiveness in bone healing remain to be investigated, these results confirmed that the simultaneous PBM and DBM treatment with or without ASCs seeding could improve the fracture repair in vivo. It suggests the probable benefit of PBM exposure to improve the outcomes of BTE treatment in large bone defects.

\section{Conclusion}

The combination of PBM and DBM with or without ASCs significantly enhanced bone healing in the CSFD in rats compared to the control, DBM alone, and ALN plus DBM groups. The simultaneous treatment with PBM and DBM with or without ASCs significantly decreased the CSFD area compared to either the solo DBM or ALN plus DBM treatments.

\section{Authors' Contribution}

MA researched data and wrote the first draft of the manuscript with the help of MK, RG, FZ, PK, AK, and RBA performed experiments. MAA, AA, FFF, and MN made suggestions. SC and $\mathrm{MB}$ edited the paper scientifically.

\section{Ethical Considerations}

The protocol of animal treatment was approved by the Animal Ethics Committee of Shahid Beheshti University (file no: IR.SBMU.MSP.REC.1397.533).

\section{Conflict of Interests}

The authors declare that they have no conflict of interest.

\section{Acknowledgments}

Demineralized bone matrix was generously donated by Hamanand Saz Baft Kish Company (TRC Corporation, Kish, Iran). 


\section{References}

1. Ignatius A, Tuckermann J. New horizons for osteoanabolic treatment? Nat Rev Endocrinol. 2018;14(9):508-9. doi:10.1038/s41574-018-0069-2

2. Lin J, Wu J, Sun S, Chen K, Wu H, Lin R, et al. Combined antisclerostin antibody and parathyroid hormone (1-34) synergistically enhance the healing of bone defects in ovariectomized rats. Z Gerontol Geriatr. 2020;53(2):163170. doi:10.1007/s00391-019-01685-2

3. Wang ML, Massie J, Perry A, Garfin SR, Kim CW. A rat osteoporotic spine model for the evaluation of bioresorbable bone cements. Spine J. 2007;7(4):466-74. doi: 10.1016/j.spinee.2006.06.400

4. Yousefzadeh N, Kashfi K, Jeddi S, Ghasemi A. Ovariectomized rat model of osteoporosis: a practical guide. EXCLI J. 2020;19:89. doi:10.17179/excli2019-1990

5. Tao Z, Zhou W, Jiang Y, Wu X, Xu Z, Yang M, et al. Effects of strontium-modified calcium phosphate cement combined with bone morphogenetic protein-2 on osteoporotic bone defects healing in rats. J Biomater Appl. 2018;33(1):3-10. doi: 10.1177/0885328218765847

6. Wu Y, Cao L, Xia L, Wu Q, Wang J, Wang X, et al. Evaluation of osteogenesis and angiogenesis of icariin in local controlled release and systemic delivery for calvarial defect in ovariectomized rats. Sci Rep. 2017;7(1):1-14. doi:10.1038/s41598-017-05392-z

7. van Houdt CI, Gabbai-Armelin PR, Lopez-Perez PM, Ulrich DJ, Jansen JA, Renno ACM, et al. Alendronate release from calcium phosphate cement for bone regeneration in osteoporotic conditions. Sci Rep. 2018;8(1):1-13. doi:10.1038/s41598-018-33692-5

8. Seeman E, Martin T. Antiresorptive and anabolic agents in the prevention and reversal of bone fragility. Nat Rev Rheumatol. 2019;15(4):225-36. doi:10.1038/s41584-0190172-3

9. Jarvis CI, Morin AK, Lynch AM. Bisphosphonates for osteoporosis prevention and treatment. Clinical Reviews in Bone and Mineral Metabolism. 2005;3(1):3-18. doi:10.1385/ BMM:3:1:003

10. Wang $\mathrm{P}$, Zhao L, Liu J, Weir MD, Zhou X, Xu HH. Bone tissue engineering via nanostructured calcium phosphate biomaterials and stem cells. Bone Res. 2014;2:14017. doi:10.1038/boneres.2014.17

11. Wu T, Yu S, Chen D, Wang Y. Bionic design, materials and performance of bone tissue scaffolds. Materials (Basel). 2017;10(10):1187. doi:10.3390/ma10101187

12. Ghamor-Amegavi EP, Yang X, Qiu J, Xie L, Pan Z, Wang J, et al. Composition control in biphasic silicate microspheres on stimulating new bone regeneration and repair of osteoporotic femoral bone defect. J Biomed Mater Res B Appl Biomater. 2020;108(2):377-90. doi:10.1002/ jbm.b.34396

13. Bae HW, Zhao L, Kanim LE, Wong P, Delamarter RB, Dawson EG. Intervariability and intravariability of bone morphogenetic proteins in commercially available demineralized bone matrix products. Spine. 2006;31(12):1299-306. doi:10.1097/01. brs.0000218581.92992.b7.

14. Zhang H, Yang L, Yang Xg, Wang F, Feng Jt, Hua Kc, et al. Demineralized bone matrix carriers and their clinical applications: an overview. Orthop Surg. 2019;11(5):725-37. doi:10.1111/os.12509

15. Shahmoradi SR, Salmani MK, Soleimanpour HR, Tavakoli $\mathrm{AH}$, Hosaini $\mathrm{K}$, Haghighipour $\mathrm{N}$, et al. Induction of chondrogenic differentiation in human mesenchymal stem cells cultured on human demineralized bone matrix scaffold under hydrostatic pressure. Tissue Eng Regen Med. 2019;16(1):69-80. doi: 10.1007/s13770-018-0164-4.

16. Chen B, Lin $\mathrm{H}$, Wang J, Zhao $\mathrm{Y}$, Wang $\mathrm{B}$, Zhao $\mathrm{W}$, et al. Homogeneous osteogenesis and bone regeneration by demineralized bone matrix loading with collagen-targeting bone morphogenetic protein-2. Biomaterials. 2007;28(6):1027-35. doi:10.1016/j. biomaterials.2006.10.013

17. Huber E, Pobloth A-M, Bormann N, Kolarczik N, SchmidtBleek K, Schell H, et al. Demineralized bone matrix as a carrier for bone morphogenetic protein-2: burst release combined with long-term binding and osteoinductive activity evaluated in vitro and in vivo. Tissue Eng Part A. 2017;23(23-24):1321-30. doi:10.1089/ten.tea.2017.0005

18. Parrilla-Almansa A, García-Carrillo N, Ros-Tárraga $\mathrm{P}$, Martínez CM, Martínez-Martínez F, Meseguer-Olmo $\mathrm{L}$, et al. Demineralized bone matrix coating Si-Ca-P ceramic does not improve the osseointegration of the scaffold. Materials (Basel). 2018;11(9):1580. doi:10.3390/ ma11091580

19. Huang Y-Z, Cai J-Q, Xue J, Chen X-H, Zhang C-L, Li $\mathrm{X}-\mathrm{Q}$, et al. The poor osteoinductive capability of human acellular bone matrix. Int J Artif Organs. 2012;35(12):10619. doi:10.5301/ijao.5000122

20. Ramis JM, Calvo J, Matas A, Corbillo C, Gayà A, Monjo M. Enhanced osteoinductive capacity and decreased variability by enrichment of demineralized bone matrix with a bone protein extract. J Mater Sci Mater Med. 2018;29(7):103. doi:10.1007/s10856-018-6115-8.

21. Schubert T, Xhema D, Vériter S, Schubert M, Behets C, Delloye $\mathrm{C}$, et al. The enhanced performance of bone allografts using osteogenic-differentiated adipose-derived mesenchymal stem cells. Biomaterials. 2011;32(34):888091. doi:10.1016/j.biomaterials.2011.08.009

22. Probst FA, Fliefel R, Burian E, Probst M, Eddicks M, Cornelsen $\mathrm{M}$, et al. Bone regeneration of minipig mandibular defect by adipose derived mesenchymal stem cells seeded tri-calcium phosphate-poly (D, L-lactide-coglycolide) scaffolds. Sci Rep. 2020;10(1):2062. doi:10.1038/ s41598-020-59038-8

23. Brennan MA, Renaud A, Guilloton F, Mebarki M, Trichet $\mathrm{V}$, Sensebé L, et al. Inferior in vivo osteogenesis and superior angiogenesis of human adipose-derived stem cells compared with bone marrow-derived stem cells cultured in Xeno-free conditions. Stem Cells Transl Med. 2017;6(12):2160-72. doi:10.1002/sctm.17-0133

24. Zhang H, Kot A, Lay YAE, Fierro FA, Chen H, Lane NE, et al. Acceleration of fracture healing by overexpression of basic fibroblast growth factor in the mesenchymal stromal cells. Stem Cells Transl Med. 2017;6(10):1880-93. doi:10.1002/sctm.17-0039

25. Caetano G, Wang W, Murashima A, Passarini JR, Bagne $\mathrm{L}$, Leite $\mathrm{M}$, et al. Tissue constructs with human adiposederived mesenchymal stem cells to treat bone defects in 
rats. Materials. 2019;12(14):2268. doi:10.3390/ma12142268

26. Orbay H, Busse B, Leach JK, Sahar DE. The effects of adipose-derived stem cells differentiated into endothelial cells and osteoblasts on healing of critical size calvarial defects. J Craniofac Surg. 2017;28(7):1874-9. doi:10.1097/ SCS.0000000000003910

27. Lin W, Xu L, Zwingenberger S, Gibon E, Goodman SB, Li G. Mesenchymal stem cells homing to improve bone healing. J Orthop Translat. 2017;9:19-27. doi:10.1016/j. jot.2017.03.002

28. Chen C, Tang Q, Zhang Y, Dai M, Jiang Y, Wang H, et al. Metabolic reprogramming by HIF-1 activation enhances survivability of human adipose-derived stem cells in ischaemic microenvironments. Cell Prolif. 2017;50(5):e12363. doi:10.1111/cpr.12363

29. Ahmadi H, Amini A, Fadaei Fathabady F, Mostafavinia A, Zare F, Ebrahimpour-Malekshah R, et al. Transplantation of photobiomodulation-preconditioned diabetic stem cells accelerates ischemic wound healing in diabetic rats. Stem Cell Res Ther. 2020;11(1):494. doi: 10.1186/s13287-02001967-2

30. Kim K, Lee J, Jang H, Park S, Na J, Myung JK, et al. Photobiomodulation enhances the angiogenic effect of mesenchymal stem cells to mitigate radiation-induced enteropathy. Int J Mol Sci. 2019;20(5):1131. doi: 10.3390/ ijms20051131

31. Peat FJ, Colbath AC, Bentsen LM, Goodrich LR, King MR. In vitro effects of high-intensity laser photobiomodulation on equine bone marrow-derived mesenchymal stem cell viability and cytokine expression. Photomed Laser Surg. 2018;36(2):83-91. doi:10.1089/pho.2017.4344

32. Min KH, Byun JH, Heo CY, Kim EH, Choi HY, Pak CS. Effect of low-level laser therapy on human adipose-derived stem cells: in vitro and in vivo studies. Aesthetic Plast Surg. 2015;39(5):778-82. doi:10.1007/s00266-015-0524-6

33. Wang Y-H, Wu J-Y, Kong SC, Chiang M-H, Ho M-L, Yeh $\mathrm{M}-\mathrm{L}$, et al. Low power laser irradiation and human adiposederived stem cell treatments promote bone regeneration in critical-sized calvarial defects in rats. PloS One. 2018;13(4):e0195337. doi:10.1371/journal.pone.0195337

34. Escudero JSB, Perez MGB, de Oliveira Rosso MP, Buchaim DV, Pomini KT, Campos LMG, et al. Photobiomodulation therapy (PBMT) in bone repair: A systematic review. Injury. 2019;50(11):1853-1867. doi:10.1016/j.injury.2019.09.031

35. Kandal S, Özmen S, Uygur S, Yagci M, Kayhan H, Elmas $\mathrm{C}$, et al. Effects of rat bone marrow-derived mesenchymal stem cells and demineralized bone matrix on cranial bone healing. Ann Plast Surg. 2016;77(2):249-54. doi:10.1097/ SAP.0000000000000274

36. Supronowicz P, Gill E, Trujillo A, Thula T, Zhukauskas R, Ramos T, et al. Human adipose-derived side population stem cells cultured on demineralized bone matrix for bone tissue engineering. Tissue Eng Part A. 2011;17(5-6):789-98. doi:10.1089/ten.TEA.2010.0357

37. Han DS, Chang HK, Park JH, Kim KR, Woo SM. Consideration of bone regeneration effect of stem cells: comparison between adipose-derived stem cells and demineralized bone matrix. J Craniofac Surg. 2014;25(1):189-95. doi:10.1097/SCS.0000000000000377

38. Schubert T, Xhema D, Vériter S, Schubert M, Behets C,
Delloye C, et al. The enhanced performance of bone allografts using osteogenic-differentiated adipose-derived mesenchymal stem cells. Biomaterials. 2011;32(34):888091. doi:10.1016/j.biomaterials.2011.08.009

39. Millett PJ, Allen MJ, Bostrom MP. Effects of alendronate on particle-induced osteolysis in a rat model. J Bone Joint Surg Am. 2002;84(2):236-49. doi:10.2106/00004623-20020200000011

40. Fridoni M, Farahani RM, Nejati H, Salimi M, Gharavi SM, Bayat M, et al. Evaluation of the effects of LLLT on biomechanical properties of tibial diaphysis in two rat models of experimental osteoporosis by a three point bending test. Lasers Med Sci. 2015;30(3):1117-25. doi:10.1007/s10103-014-1706-1

41. Asgari M, Gazor R, Abdollahifar MA, Fathabady FF, Zare F, Norouzian M, et al. Combined therapy of adiposederived stem cells and photobiomodulation on accelerated bone healing of a critical size defect in an osteoporotic rat model. Biochem Biophys Res Commun. 2020;530(1):173180. doi: 10.1016/j.bbrc.2020.06.023.

42. Zhu Y, Liu T, Song K, Fan X, Ma X, Cui Z. Adipose derived stem cell: a better stem cell than BMSC. Cell Biochemistry and Function: Cellular biochemistry and its modulation by active agents or disease. Cell Biochem Funct. 2008;26(6):664-75. doi:10.1002/cbf.1488

43. Struck MB, Andrutis KA, Ramirez HE, Battles AH. Effect of a short-term fast on ketamine-xylazine anesthesia in rats. J Am Assoc Lab Anim Sci. 2011;50(3):344-8.

44. Mostafavinia A, Farahani RM, Abbasian M, Farahani MV, Fridoni MJ, Zandpazandi S, et al. Effect of pulsed wave low-level laser therapy on tibial complete osteotomy model of fracture healing with an intramedullary fixation. Iran Red Crescent Med J. 2015;17(12):e32076. doi: 10.5812/ ircmj.32076

45. Gupta A KP. Assessment of the histological state of the healing wound. Plast Aesthet Res. 2015;2(2):239-42. doi:10.4103/2347-9264.158862

46. Hamada T, Matsubara H, Yoshida Y, Ugaji Sh, Nomura I, Tsuchiya H. Autologous adipose-derived stem cell transplantation enhances healing of wound with exposed bone in a rat model. PLoS One. 2019; 14(5): e0214106. doi: 10.1371/journal.pone.0214106

47. Ciani O, Armeni P, Boscolo PR, Cavazza M, Jommi C, Tarricone R. De innovatione: The concept of innovation for medical technologies and its implications for healthcare policy-making. Health Policy Technol. 2016;5(1):47-64. doi:10.1016/j.hlpt.2015.10.005

48. Pang L, Shen Y, Hu H, Zeng X, Huang W, Gao H, et al. Chemically and physically cross-linked polyvinyl alcoholborosilicate gel hybrid scaffolds for bone regeneration. Mater Sci Eng C Mater Biol Appl. 2019;105:110076. doi:10.1016/j.msec.2019.110076

49. Leppik L, Zhihua H, Mobini S, Parameswaran VT, EischenLoges M, Slavici A, et al. Combining electrical stimulation and tissue engineering to treat large bone defects in a rat model. Sci Rep. 2018;8(1):1-14. doi:10.1038/s41598-01824892-0

50. Seebach C, Schultheiss J, Wilhelm K, Frank J, Henrich D. Comparison of six bone-graft substitutes regarding to cell seeding efficiency, metabolism and growth behaviour of 
human mesenchymal stem cells (MSC) in vitro. Injury. 2010;41(7):731-8. doi:10.1016/j.injury.2010.02.017

51. Sohn H-S, Oh J-K. Review of bone graft and bone substitutes with an emphasis on fracture surgeries. Biomater Res. 2019;23(1):9. doi:10.1186/s40824-019-0157-y

52. Tang D, Ju C, Liu Y, Xu F, Wang Z, Wang D. Therapeutic effect of icariin combined with stem cells on postmenopausal osteoporosis in rats. J Bone Miner Metab. 2018;36(2):180-8. doi:10.1007/s00774-017-0831-x

53. Pavone V, Testa G, Giardina S, Vescio A, Restivo DA, Sessa G. Pharmacological therapy of osteoporosis: a systematic current review of literature. Front Pharmacol. 2017;8:803. doi:10.3389/fphar.2017.00803

54. Sellmeyer DE. Atypical fractures as a potential complication of long-term bisphosphonate therapy. JAMA. 2010;304(13):1480-4. doi:10.1001/jama.2010.1360

55. Kennel KA, Drake MT. Adverse effects of bisphosphonates: implications for osteoporosis management. Mayo Clin Proc. 2009;84(7):632-7; quiz 638. doi: 10.1016/S00256196(11)60752-0.

56. Casado-Díaz A, Dorado G, Giner M, Montoya MJ, NavarroValverde C, Díez-Pérez A, et al. Proof of Concept on Functionality Improvement of Mesenchymal Stem-Cells, in Postmenopausal Osteoporotic Women Treated with Teriparatide (PTH1-34), After Suffering Atypical Fractures. Calcif Tissue Int. 2019;104(6):631-40. doi:10.1007/s00223019-00533-0

57. Papapetrou PD. Bisphosphonate-associated adverse events. Hormones (Athens). 2009;8(2):96-110. doi:10.14310/ horm.2002.1226

58. Ma T, Sun J, Zhao Z, Lei W, Chen Y, Wang X, et al. A brief review: adipose-derived stem cells and their therapeutic potential in cardiovascular diseases. Stem Cell Res Ther. 2017;8(1):124. doi:10.1186/s13287-017-0585-3

59. Yoshida Y, Matsubara H, Fang X, Hayashi K, Nomura I, Ugaji S, et al. Adipose-derived stem cell sheets accelerate bone healing in rat femoral defects. PloS One. 2019;14(3):e0214488. doi:10.1371/journal.pone.0214488

60. Jin Y-Z, Lee JH. Mesenchymal stem cell therapy for bone regeneration. Clin Orthop Surg. 2018;10(3):271-8. doi:10.4055/cios.2018.10.3.271

61. Kloeters O, Berger I, Ryssel H, Megerle K, Leimer U, Germann G. Revitalization of cortical bone allograft by application of vascularized scaffolds seeded with osteogenic induced adipose tissue derived stem cells in a rabbit model. Arch Orthop Trauma Surg. 2011;131(10): 1459-66. doi:10.1007/s00402-011-1306-5

62. Levi B, James AW, Nelson ER, Vistnes D, Wu B, Lee M, et al. Human adipose derived stromal cells heal critical size mouse calvarial defects. PloS One. 2010;5(6):e11177. doi:10.1371/journal.pone.0011177

63. Wu Y, Chen L, Scott PG, Tredget EE. Mesenchymal stem cells enhance wound healing through differentiation and angiogenesis. Stem Cells. 2007;25(10):2648-59. doi:10.1634/ stemcells.2007-0226

64. Seo Y, Shin T-H, Kim H-S. Current strategies to enhance adipose stem cell function: an update. Int J Mol Sci. 2019;20(15):3827. doi:10.3390/ijms20153827

65. Skondra FG, Koletsi D, Eliades T, Farmakis ETR. The effect of low-level laser therapy on bone healing after rapid maxillary expansion: a systematic review. Photomed Laser Surg . 2018;36(2):61-71. doi:10.1089/pho.2017.4278

66. dos Santos Santinoni C, Oliveira HFF, de Souza Batista VE, Lemos CAA, Verri FR. Influence of low-level laser therapy on the healing of human bone maxillofacial defects: A systematic review. J Photochem Photobiol B. 2017;169:83-9. doi: 10.1016/j.jphotobiol.2017.03.004

67. Choi K, Kang BJ, Kim H, Lee S, Bae S, Kweon OK, et al. Low-level laser therapy promotes the osteogenic potential of adipose-derived mesenchymal stem cells seeded on an acellular dermal matrix. J Biomed Mater Res B Appl Biomater. 2013;101(6):919-28. doi: 10.1002/jbm.b.32897

68. Bosco AF, Faleiros PL, Carmona LR, Garcia VG, Theodoro LH, de Araujo NJ, et al. Effects of low-level laser therapy on bone healing of critical-size defects treated with bovine bone graft. J Photochem Photobiol B. 2016;163:303-10. doi:10.1016/j.jphotobiol.2016.08.040

69. Cunha MJ, Esper LA, Sbrana MC, de Oliveira PG, do Valle AL, de Almeida ALP. Effect of low-level laser on bone defects treated with bovine or autogenous bone grafts: in vivo study in rat calvaria. Biomed Res Int. 2014;2014:104230. doi:10.1155/2014/104230

70. Fernandes K, Magri A, Kido H, Parisi J, Assis L, Fernandes K, et al. Biosilicate/PLGA osteogenic effects modulated by laser therapy: In vitro and in vivo studies. J Photochem Photobiol B. 2017;173:25865. doi:10.1016/j.jphotobiol.2017.06.002 